\title{
Osteochondral lesions of the talus
}

\author{
G. M. M. J. Kerkhoffs ${ }^{1,2,3} \cdot$ J. Karlsson ${ }^{4}$
}

Published online: 10 August 2019

(c) European Society of Sports Traumatology, Knee Surgery, Arthroscopy (ESSKA) 2019

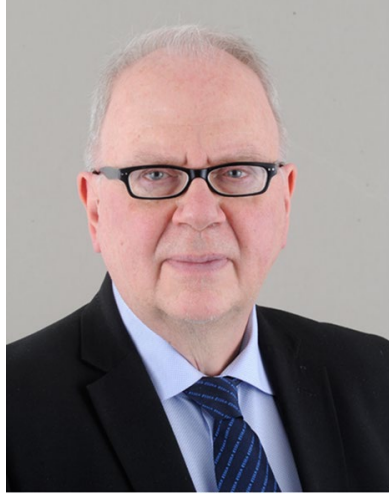

J. Karlsson

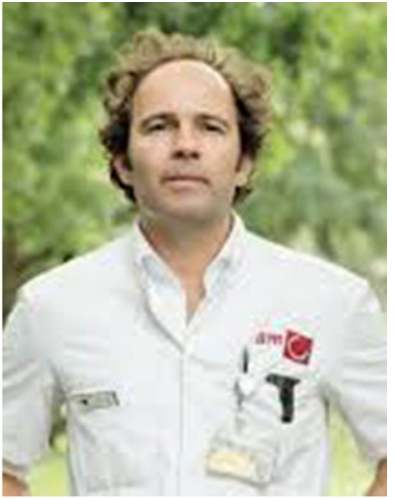

G. M. M. J. Kerkhoffs
Osteochondral lesions of the talus (OCLT) are defined by damage to the talar articular cartilage and its subchondral bone. OCLT present a common ankle pathology and have been shown to occur in over $65 \%$ of chronic ankle sprains and up to $75 \%$ of ankle fractures [4, 5, 9]. An ankle sprain or fracture may cause a cartilage injury as the talar dome impacts on the distal tibial plafond. This may initiate the development of an osteochondral cyst through cracks in the subchondral plate, destabilise a previously stable osteochondral lesion or create an osteochondral fracture, leading to chronic deep ankle pain and disability in the medium to long

$\triangle$ J. Karlsson

jon.karlsson@telia.com

1 Department of Orthopedic Surgery, Amsterdam Movement Sciences, Amsterdam University Medical Centers, Amsterdam, The Netherlands

2 Academic Center for Evidence Based Sports Medicine (ACES), Amsterdam, The Netherlands

3 Amsterdam Collaboration for Health and Safety in Sports (ACHSS)AMC/VUmc IOC Research Center, Amsterdam, The Netherlands

4 Department of Orthopaedics, Sahlgrenska University Hospital, Sahlgrenska Academy, Gothenburg University, Gothenburg, Sweden term. In addition, possible genetic predisposition has been discussed as a possible cause of OCLT. The injuries may have a severe impact on the quality of life of active patients, due to deep ankle pain during weight-bearing and sporting activities. OCLT has the potential to progress into ankle joint osteoarthritis, and it is known that the vast majority of ankle joint osteoarthritis is posttraumatic [9].

Management of OCLT is not yet able to guarantee a successful return to pain-free weight-bearing and sporting activities. Non-surgical management, including physiotherapy, injection therapy, non-weight-bearing periods and immobilisation, may relieve symptoms temporarily, but seldom lead to a persistent return to sporting activities, however. Even in paediatric OCLT, a period of non-surgical treatment is known to lead to limited success. At the same time, it is also a fact that surgical treatment after a period of non-surgical management provides a higher success rate [14].

Surgical management of OCLT is dependent on a variety of aspects of the patient and the lesion: limb alignment, ankle joint instability, the size, location and morphological characteristics of OCLT (i.e. occurrence of cysts) and failed previous surgical treatment(s). For small primary defects, first-line surgical management options that are frequently applied consist of (arthroscopic) fixation, bone marrow stimulation and possibly retrograde drilling [3, 10]. Fixation is preferred because first it is proven that subchondral bone healing after fixation of an osteochondral talar defect is superior compared with bone marrow stimulation [3, 10, 13] and second bone marrow stimulation is still possible after a failed fixation. It appears from the recent study of Becher et al. that the addition of an acellular matrix to a bone marrow stimulation does not lead to a better result [1]. This study compared the results of arthroscopic microfracture vs. arthroscopic autologous matrix-induced chondrogenesis using a collagen I/III matrix (AMIC) and concluded that for defects as treated in this study, it is not beneficial to add the collagen I/III matrix to the microfracture procedure [1]. In their study, the Kennedy group showed that the overall return to play rate after bone marrow stimulation is very 
acceptable and it might be recommended to start weightbearing early in the rehabilitation process [7].

In the case of large cystic defects or in the case of failure of first-line surgical treatment, fixation is not an option anymore, and more extensive and invasive surgery can be considered a necessary step in the management process. Treatment strategies that are currently frequently used are osteochondral allograft procedures, osteochondral autograft transfer system (OATS) procedures and matrix-associated chondrocyte implantation (ACI, MACI) [11]. Even though the OATS procedure is regarded as an effective surgical management option with success rates up to $90 \%$ reported in the literature, donor-site morbidity has been reported to occur relatively frequently with incidence rates ranging from 11 to $35 \%$ when the graft was harvested from the ipsilateral femoral condyle $[3,11]$. As an alternative to an OATS procedure harvesting the graft from the knee, an autologous osteoperiosteal graft can be harvested from the iliac crest [2, $6,8]$. Matrix-associated stem cell transplantation (MAST) is a good alternative treatment that does not have the limitation of donor-site morbidity. Murphy et al. describe the results of a single-surgeon cohort for larger lesions, i.e. $15 \mathrm{~mm}^{2}$ or more and/or failed alternative treatment(s) [12]. MAST demonstrated encouraging results in lesions which prove challenging to treat, even after a "failed microfracture" [12].

Future management should probably aim for primary preventive interventions to prevent osteochondral lesions to develop from initial posttraumatic cracks in the cartilage by cushioning the lesions. Thereafter as second-stage treatment, optimization of fixation and bone marrow stimulation techniques - for example with the use of MAST — will allow a higher number of patients who are able to return to pain-free sports activities.

The future of OCLT treatment will be focused on an individualised approach, taking into account all patient characteristics and OCLT morphological aspects, with a keen focus on the three requirements for optimal tissue engineering: a source of cells, a scaffold, and local growth factors. We expect that the articles published in the current $[1,7,12]$ and upcoming issue(s) of the KSSTA journal will stimulate further debate, research and ultimately improve the management for this common and troublesome sport-related injury in the near future.

\section{References}

1. Becher C, Malahias MA, Mahmoud-Ali M, Maffulli N, Thermann H (2019) Arthroscopic Microfracture vs. Arthroscopic
Autologous Matrix-Induced Chondrogenesis for the Treatment of Articular Cartilage Defects of the Talus. Knee Surg Sports Traumatol Arthrosc. https://doi.org/10.1007/s00167-018-5278-7

2. Chen W, Tang K, Yuan C, Zhou Y, Tao X (2015) Intermediate results of large cystic medial osteochondral lesions of the talus treated with osteoperiosteal cylinder autografts from the medial tibia. Arthroscopy 31:1557-1564

3. Dahmen J, Lambers KTA, Reilingh M, van Bergen CJA, Stufkens SAS, Kerkhoffs GMMJ (2018) No superior treatment for primary osteochondral defects of the talus. Knee Surg Sports Traumatol Arthrosc 26(7):2142-2157

4. Hintermann B, Boss A, Schafer D (2002) Arthroscopic findings in patients with chronic ankle instability. Am J Sports Med 30:402-409

5. Hintermann B, Regazzoni P, Lampert C, Stutz G, Gachter A (2000) Arthroscopic findings in acute fractures of the ankle. J Bone Joint Surg Br 82(3):345-351

6. Hu Y, Guo Q, Jiao C, Mei Y, Jiang D, Wang J, Zheng Z (2013) Treatment of large cystic medial osteochondral lesions of the talus with autologous osteoperiosteal cylinder grafts. Arthroscopy 29:1372-1379

7. Hurley ET, Shimozono Y, Myerson CL, McGoldrick NP, Yasui Y, Kennedy JG (2019) High Reported Rate of Return to Play Following Bone Marrow Stimulation for Osteochondral Lesions of the Talus. Knee Surg Sports Traumatol Arthrosc. https://doi. org/10.1007/s00167-018-4913-7

8. Kerkhoffs GM, Stufkens SAS, Dahmen J, Altink JN (2019). Talar Osteo-Periostic grafting from the Iliac Crest (TOPIC): 1-year results of a new press-fit surgical treatment for large talar osteochondral defects. Abstract. International Congress on Cartilage Repair of the Ankle, March 2019, Dublin, Ireland

9. Kerkhoffs GM, Kennedy JG, Calder JD, Karlsson J (2016) There is no simple lateral ankle sprain. Knee Surg Sports Traumatol Arthrosc. 24(4):941-943

10. Kerkhoffs GM, Reilingh ML, Gerards RM, de Leeuw PA (2016) Lift, drill, fill and fix (LDFF): a new arthroscopic treatment for talar osteochondral defects. Knee Surg Sports Traumatol Arthrosc. 24(4):1265-1271

11. Lambers KTA, Dahmen J, Reilingh ML, van Bergen CJA, Stufkens SAS, Kerkhoffs GMMJ (2018) No superior surgical treatment for secondary osteochondral defects of the talus. Knee Surg Sports Traumatol Arthrosc. 26(7):2158-2170

12. Murphy EP, Fenelon C, Egan C, Kearns SR (2019) Matrix-associated stem cell transplantation is successful in treating talar osteochondral lesions. Knee Surg Sports Traumatol Arthrosc. https:// doi.org/10.1007/s00167-019-05452-z

13. Reilingh ML, Lambers KTA, Dahmen J, Opdam KTM, Kerkhoffs GMMJ (2018) The subchondral bone healing after fixation of an osteochondral talar defect is superior in comparison with microfracture. Knee Surg Sports Traumatol Arthrosc. 26(7):2177-2182

14. Reilingh ML, Kerkhoffs GM, Telkamp CJ, Struijs PA, van Dijk CN (2014) Treatment of osteochondral defects of the talus in children. Knee Surg Sports Traumatol Arthrosc. 22(9):2243-2249

Publisher's Note Springer Nature remains neutral with regard to jurisdictional claims in published maps and institutional affiliations. 